\title{
SNS and the Lived Experiences of Queer Youth
}

\author{
Tommaso Armstrong \\ Tommaso.Armstrong@student.uts.edu.au \\ University of Technology Sydney \\ Ultimo, NSW, Australia
}

\author{
Tuck Wah Leong \\ TuckWah.Leong@uts.edu.au \\ University of Technology Sydney \\ Ultimo, NSW, Australia
}

\begin{abstract}
Technology design has not adequately included a queer perspective, even though digital technologies such as social networking sites (SNS) have been shown to play vital roles in the lives and well-being of queer people. SNS provide queer people with a means to explore their identities, learn about queerness and connect to others with similar experiences. However, SNS use can also have detrimental effects, exposing queer people to harm and victimisation. To date, there is not much effort in $\mathrm{HCI}$ to understand the experiences of queer people with SNS. As a result, we lack understanding of how SNS and other social technologies could be designed in ways that are supportive of queer people's well-being. The findings from this exploratory study reveal how particular digital technologies can have complex effects in shaping queer people's experiences and their well-being.
\end{abstract}

\section{CCS CONCEPTS}

- Human-centered computing $\rightarrow$ Empirical studies in $\mathrm{HCI}$; - Social and professional topics $\rightarrow$ Sexual orientation.

\section{KEYWORDS}

queer, LGBTQIA+, lived experiences, SNS

\section{ACM Reference Format:}

Tommaso Armstrong and Tuck Wah Leong. 2019. SNS and the Lived Experiences of Queer Youth. In 31ST AUSTRALIAN CONFERENCE ON HUMANCOMPUTER-INTERACTION (OZCHI'19), December 2-5, 2019, Fremantle, WA, Australia. ACM, New York, NY, USA, 5 pages. https://doi.org/10.1145/ 3369457.3369497

\section{INTRODUCTION}

This paper presents experiences of queer people and how the use of social networking sites (SNS) can shape their experiences. Following [10], our use of "queer" refers to, "anyone who does not identify as exclusively heterosexual (including gay, lesbian, bisexual, pansexual, asexual, transgender, genderqueer, questioning, and others)". Like [10,14], we explore the experiences of queer youth between the ages of 18-28, a demographic that grew up with SNS during the formative years of their identity development as queer. This research is important to HCI because to date, there's been little effort to understand how technology use shapes the experiences

Permission to make digital or hard copies of all or part of this work for personal or classroom use is granted without fee provided that copies are not made or distributed for profit or commercial advantage and that copies bear this notice and the full citation on the first page. Copyrights for components of this work owned by others than ACM must be honored. Abstracting with credit is permitted. To copy otherwise, or republish, to post on servers or to redistribute to lists, requires prior specific permission and/or a fee. Request permissions from permissions@acm.org.

OZCHI'19, December 2-5, 2019, Fremantle, WA, Australia

(c) 2019 Association for Computing Machinery.

ACM ISBN 978-1-4503-7696-9/19/12 . \$15.00

https://doi.org/10.1145/3369457.3369497 of the queer people. Despite queer people making up 5-10\% of the population [21], a search of the ACM Digital Library on July 29, 2019 , for keywords or paper titles that engage with queer people or queer theory, revealed only 103 results out of $2,800,000+$ records. There were $70 \%$ more results that pertained to the fact that an author's name was "Gay". Thus, we argue that queer people are not only marginalised in society but also within HCI, where the focus and efforts are overwhelming heteronormative.

The need to include queer perspectives in $\mathrm{HCI}$ has been championed by a few researchers who have called for efforts to be more inclusive of diverse experiences and perspectives. [12] argues that conservatism and technocentrism within $\mathrm{HCI}$ threaten to reinforce problematic status quos through a desire to be apolitical or ahistorical. Similarly, [1] warns of the dangers of universalist design which, "demotes cultural, social, regional, and national differences in user experiences and outlooks" and argues that more pluralist design which accounts for the complexity of human experience will likely provide more human-centred outcomes.

Our literature review found that research around the queer community to date has primarily focused on their use of specific technology, e.g. Grindr, a dating app, and particular sub-groups, e.g. gay men. However, SNS are not used in isolation and the focus on particular sub-groups has left others underrepresented. Thus, our exploratory study takes a broader interest in exploring holistically how use of SNS plays a role in shaping the experiences and sense of well-being of a diverse group of queer people.

\section{RELATED WORK}

There has been a growing interest to understand better how digital technologies, particularly SNS, can affect well-being. Research within HCI and psychology has shown how SNS, like a doubleedged sword, can have both positive and negative effects depending on how they are used $[3,7,8,23]$. While there are clear benefits afforded by SNS use, the way SNS negatively impacts people's well-being is only just starting to be understood.

While research shows how adverse mental health outcomes can arise from SNS usage [3, 7, 9, 23], we argue that these adverse outcomes may be heightened for queer people. This is due to the additional burden of minority stress faced by queer people. Minority stress is brought about by the stigmatisation of their identities, arising from a range of sources, including homophobic environments, victimisation, learned expectations of rejection and the psychological burden of concealing one's identity $[15,18]$.

Research shows that SNS use by queer people can lead to this minority stress, with them potentially experiencing victimisation, discrimination, or being inadvertently outed [6, 11, 14, 19]. For example, queer youth are 2-3x more likely to be the targets of cyber-bullying [14]. [6] argue that while queer people can benefit 
from feeling safe enough to be visibly queer online, SNS don't afford enough control over "selective visibility" which might allow only certain trusted friends to see queer aspects of one's identity. Similarly, the convergence, particularly on Facebook, of connections from multiple contexts which often mirror real-life networks, can lead to problematic and unintended disclosure or outing as a result of context collapse, described as the "flatten[ing of] multiple audiences into one" [13]. While some SNS, including Facebook, allow the option to restrict the visibility of specific posts, interacting with content on the site often creates traces over which there are minimal privacy controls. For example, RSVPing to a queer event might automatically create a post which announces this interaction to one's entire Facebook network [6]. Even when the individual is not the direct target of harm, they may experience adverse effects, for example, in situations where one bears witness to anti-queer discourse or abuse aimed at others [19]. Harassment from outsiders is not the only issue, though. Research concerning trans safe spaces found that harm is often perpetuated by people in one's social circles in what the authors refer to as "lateral violence" or "insider harm" [19].

On the other hand, SNS have been shown to support queer people by allowing for social support and learning. [10] show that queer youth benefit significantly from the social support that SNS can afford them. This social support, often from peers or role-models, enables them to feel less isolated and provides a way to explore and learn how to enact their identities with others. This is particularly beneficial because queer people have to make sense of and allow for the emergence of their identities in a way that cis-gender heterosexuals (cis-hets) do not [10]. Furthermore, social support can act as a protective factor against minority stress [18], contributing positively to well-being. These interactions are particularly vital when individuals don't have existing relationships with supportive figures or peers [14], especially for those with identities that are less represented in society (e.g. asexual or transgender) [10].

Anonymity and privacy within online queer spaces is a significant priority when there is such a high propensity for harm. The anonymity afforded by the internet has made it an essential tool for queer individuals to connect with other queer people [19]. Historically, congregating in physical spaces has caused safety concerns for queer people., As a result, much social activity has now shifted online [22]. Feelings of anonymity are also crucial in the early stages of developing one's identity due to the commonly experienced fear of stigmatisation if one were to be discovered [10]. The importance of anonymity can be seen in research about the queer community on Tumblr. [4] notes how the anonymity afforded by the site enables greater honesty and gives queer users a space to experiment with their identity. In addition, anonymity affords users the ability to have less specific or static representations of their identity. This is particularly supportive of gender-diverse individuals who may have emergent or fluid gender identities $[5,11]$.

A range of dating apps, which we include in the scope of SNS, are increasingly becoming a popular space for queer people to interact. However, our review of the literature shows that Grindr, a location-based dating application for queer men, is the main app to have received attention from researchers [2, 17, 22]. While Grindr is often perceived as a place to find casual sex, many people use it in the hopes of finding social connections or romantic relations
Table 1: Participant details

\begin{tabular}{ccc}
\hline Participant \# & Age & Queer Identity \\
\hline 1 & 22 & Bisexual \& transgender \\
2 & 23 & Gay \\
3 & 26 & Gay \\
4 & 18 & Bisexual, possibly non-binary trans \\
5 & 19 & Bisexual/queer \\
6 & 24 & Gay \\
7 & 28 & Gay \\
8 & 25 & Queer, bisexual, pansexual \\
9 & 18 & Transgender \\
\hline
\end{tabular}

[22]. Researchers have noted, especially amongst gay men, how dating apps are increasingly replacing offline queer spaces such as bars due to the convenience and anonymity they afford when seeking connection to others [17]. Alluded to by [22], apps such as Grindr can also be places where lateral violence can prosper through harassment and abuse perpetrated by others on the platform.

While some within HCI have explored the experiences of queer people, the SIG on Queer(ing) HCI in 2019 [20] has identified several gaps in the literature. Empirical work has focused predominantly on dating and disclosure contexts - a small part of the queer experience. Additionally, the subjects of studies have disproportionally been cis-gay men. Another limitation identified in technology-related queer research is the focus placed on information seeking uses or sexual health over the more social contexts of online spaces $[10,14,22]$. Furthermore, many studies focus on a single technology or SNS, such as Facebook (e.g. [14]) and Grindr (e.g. [22]), when in actuality, queer people often use a combination of technologies.

\section{METHOD}

\subsection{Participants}

We recruited participants through social media posts on Facebook and Twitter shared publicly, as well as in secret Facebook groups of Queer Collectives at local universities. We directed respondents to a survey that collected data regarding their sexual and gender identity as well as age. In collecting this data, we aimed to ensure a broad cross-section of queer identities among participants. We also collected contact details so that we could contact them if they met the selection criteria. We should note that the survey was itself a target of homophobic harassment in the form of fake responses with offensive language. It highlighted to us, the level of homophobia that is present in daily life. A summary of participants is presented in Table 1.

\subsection{Unstructured Interviews}

The exploratory nature of this study meant that we chose unstructured interviews to learn about our participants' experiences. Topics of the interview included participants' identity/queerness, cultural/religious background, familial and social relationships, use of technology and attitudes towards it (particularly social platforms), well-being and work/financial situation. The first author 
conducted the interviews and used his queerness to position himself as an insider to build rapport with participants and make them feel comfortable. The interviews were transcribed from recordings and analysed using thematic analysis [16] leading to the development of themes, which will we will describe in the next section.

\section{FINDINGS}

The following section presents a subset of themes that emerged in the analysis of the interview data. Collectively, they reveal the lived experiences of queerness and the different ways technology shape queer experiences. The themes are presented in an order that reflects the chronology of participants' journeys from self-discovery and acceptance, to living in the closet, coming out and life beyond. While they follow a typical journey based on experiences gathered, we note that these stages may overlap, be experienced in a different order or not at all.

\subsection{Technologies for Self-Discovery}

All participants had a moment of realisation that they were queer. This was either by realising that not everyone had the same nonheteronormative feelings/attractions as them, or, by making sense of, and accepting, feelings/attractions they had that weren't normative. Online spaces provided a place where they could explore, or even discover these identities, often with the safety afforded by being able to visit these communities privately and anonymously. This avenue for exploration was particularly important where participants didn't have access to existing access to queer role models.

The most commonly mentioned platform for this was Tumblr, where participants found queer communities with whom they could engage. However, participants used a range of SNS. P4 and P9 were both members of a Facebook group aimed at queer school-aged kids in their state. However, due to the privacy settings of the group, they had to be invited by existing members they had come out to. P6, at the age of 17, found an online forum for gay teens while browsing the internet and became an active member of the community and eventually a moderator. He spoke of how valuable it was to learn from his peers on that site and how the ability to express his queerness openly, gave him the confidence to come out.

In addition to SNS, participants also used online resources for exploration and learning. These resources were particularly useful surrounding topics they wouldn't otherwise be exposed to, e.g. sexual education, queer relationships or queer history. For example, P7 learned about local queer history online and felt a greater sense of belonging with the community.

\subsection{Life in the Online Closet}

All participants underwent a period where they had realised that they were queer but felt unready, reluctant or even unsafe to come out. This period was talked about by most participants as quite a difficult time that involved feeling unable to express themselves and living double lives, especially when interacting with their families.

Participants described going to great lengths to hide their queerness from family, both in the ways that they used technology and in the physical world. Multiple spoke of how they refrained from liking content or RSVPing to events on Facebook in case the digital traces these actions would cause were seen by those they weren't out to. P4 talked about how their online self is not their true self out of safety concerns. In the physical world, participants spoke about trying to appear straight, hiding friendships with visibly queer people from their families and not going to major queer events in case their parents correlated the dates. P4 who is closeted to their family described feeling like a "secret spy".

\subsection{Coming Out through Technology}

Many of the participants came out progressively and/or strategically, often starting with friends that were closest or most likely to be accepting. Sometimes this was an explicit conversation, message or Facebook post, but often it was more subtle hints at their queerness. For example, saying something about someone of the same gender being attractive. Often the reaction to them coming out was overwhelmingly positive despite the fear that preceded it although some participants did have negative experiences. After telling those close to him and his family, P6 used Facebook to broadcast his queerness. He saw it as a way to get the process of coming out over with quickly, and to avoid the stress of having to keep a mental log of who he was out to.

\subsection{Technology and Encounters with Heteronormativity}

Many participants still feel the burden of actively managing their self-expression in certain situations despite having come out to friends and family. In unfamiliar contexts, especially professional ones, participants spoke of being cautious about being perceived as queer until they were sure they would be safe. For example, P6 spoke about not revealing he was gay at a new job until he saw that a visibly queer colleague was treated well. P4 uses Facebook to vet potential friends or acquaintances. If someone's profile offers anti-queer or alt-right sentiments, they will refuse to befriend them out of safety concerns. For P9, the inability to restrict the privacy of specific posts on Instagram combined with the social pressure to accept follow requests from his colleagues has been particularly troubling. He doesn't want to delete the posts he made pre-transition that would expose him as trans, nor does he want his colleagues to see them. The result is a stressful situation that he doesn't know how to escape.

Many felt the continual burden of having to explain their identity or actions to cis-hets, especially where their queer identity was more complex than merely being gay. Most of the participants had predominantly queer friends and many ascribed the emotional labour of having to explain themselves as a significant reason. Multiple participants used different labels to simplify their identity when talking to cis-hets as they felt this would ease their interactions and result in fewer questions. For P1 this meant conforming to presenting themselves in a trans-masculine way amongst those to whom they had come out to as a trans man, despite having a fluid gender identity that often didn't reflect their earlier declaration. P5 and P9 preferred to refer to themselves broadly as being "queer" however often identified themselves as bi-sexual. For those that were attracted to more than one gender, they described having to continually remind people they were queer when they were in "straight" relationships. 


\subsection{Seeking Queer Spaces}

This need to explain themselves drove most participants to have mainly queer friends but also to seek out safe physical and virtual spaces where they felt more comfortable being themselves and could let their guard down. When talking about queer-friendly physical spaces, dedicated "Queer Spaces" on university campuses and queer bars/clubs were the most frequently mentioned by our participants. In the virtual realm, participants spoke mainly of their experiences within Facebook groups and on dating apps.

While these spaces provide an opportunity to escape heteronormativity, navigating these spaces often presented additional challenges to participants. $\mathrm{P} 4$ spoke of how queer spaces allow them to express themselves freely, while others spoke of cultures of exclusion and harassment that often plagued these spaces. Many of our participants expressed a desire for more inclusive and less sexualised queer spaces. They mentioned places to hang out other than clubs or places to find friendship online instead of dating apps.

Some participants were drawn to dating apps seeking connection to others that went beyond finding romantic or sexual partners. Experiences varied considerably across different apps in terms of how positive they were. Tinder was touted as being much more civil and dating-centric compared to Grindr. Participants saw the latter as both toxic and much more sexualised. However, despite the various negative experiences on Grindr, they continue to be drawn to the app in the hopes of finding positive experiences.

The five participants who had used Grindr described a plethora of negative experiences on it. These ranged from receiving abusive or unsolicited explicit messages to seeing profiles that had racist or otherwise discriminatory lists of unattractive characteristics. They detailed a culture that idolises people who look a certain way and ignores or actively rejects those that don't conform. This negatively impacted their feelings of self-worth. Both P6 and P7 explained how they see the culture on Grindr as self-reinforcing. They described how new users to the app are initially submitted to this toxic culture and eventually become bitter enough to perpetuate it themselves. Participants partly blame the anonymity afforded by the platform for dehumanising profiles and allowing people not to be held accountable for their actions.

By contrast, participants found Tinder to be a friendlier space. Whereas on Grindr anyone can start a conversation, on Tinder both parties need to have "liked" the other. Participants credited this difference for significantly reducing the number of abusive messages they received. It also lowered the potential for them to be rejected as anyone they messaged had to have already liked them.

While Grindr doesn't require disclosure of gender when creating a profile, Tinder does. This is because Tinder relies on people to report their gender and the gender(s) of those they want to see to determine the profiles they are shown. In doing this, it assumes a gender binary which proves problematic for $\mathrm{P} 1$ who is non-binary. To overcome this limitation, P1 alternates the gender that they report. They also change the gender of those they want to see to match to ensure that they are only shown to other queer people.

\section{DISCUSSION}

Despite growing acceptance, queer people still face significant discrimination. All participants continue to face some homophobia, have to manage their self-expression due to fears around safety and feel more comfortable around other queer people. While some digital technologies have helped mitigate some of these challenges, it is clear that at the same time, it has the potential to open queer people up to harm.

Like others $[5,10,14]$, our participants often turned to online spaces to connect and learn about their identity while they were in the formative stages of their queer identity. $[5,10]$ noted that people often outgrew or decided to move on from these communities once they had developed a greater sense of self-acceptance, something we also saw in our findings. However, despite moving on from these platforms, our participants continue to express the desire for connection to others, including struggling to find alternative spaces (something not discussed by $[5,10]$ ).

As noted previously, five participants turned to Grindr seeking friends, but found a culture that they perceived to have negatively impacted their psychological well-being. $[15,18]$ cite the psychological burden of always expecting rejection based on one's queerness as a cause for mental health disparities. Our participants' experiences on Grindr reveal a strikingly similar picture of rejection and its negative impacts, but instead, from within their own community. While others $[2,22]$ have looked at the different motivations for the use of Grindr and explored reasons for leaving, prior work has only alluded in passing to its toxic culture of abuse and harassment. Likewise, while lateral violence and toxic cultures within the queer community have been discussed in specific contexts, e.g. within trans safe spaces [19] and on Tumblr [5], its pervasiveness is clearly an issue which requires further exploration.

Our work, like others $[6,11,12,19]$, found a need for greater autonomy over self-presentation on SNS. Some platforms, such as Tumblr, allow a great deal of autonomy due to the anonymity they afford [5]. However, similarly to [19], we found that, while a strategy for enabling autonomy, anonymity can enable harassment and victimisation. SNS that don't offer anonymity or provide adequate autonomy to manage self-presentation, constrain participants in the way they can behave and the vigilance that this requires likely contributes to minority stress. As such, we echo the calls of $[6,11$, 12] to design SNS that allow queer people greater autonomy over the way their identity and actions are presented. In doing this, we agree with [19] when they argue for designers to "examine the very structures of their technological creations and the way they enable harm against marginalized individuals".

\section{LIMITATIONS}

Our exploratory study adds to the slowly growing scholarship by adding further nuanced understandings of how digital technology use shape the experiences and well-being of queer people. However, this understanding is limited by the small sample size. Also, despite seeking participants with diverse queer identities, no one identified as intersex, asexual or lesbian, although there were multiple bisexual non-cis-men. This disparity will need to be addressed in future work. Nevertheless, this paper provides a window into the complex experiences that queer people face and highlights the need for further work in this space. 


\section{REFERENCES}

[1] Shaowen Bardzell. 2010. Feminist HCI: Taking Stock and Outlining an Agenda for Design. (2010), 10.

[2] Jed R Brubaker, Mike Ananny, and Kate Crawford. 2016. Departing glances: A sociotechnical account of 'leaving' Grindr. New Media \& Society 18, 3 (March 2016), 373-390. https://doi.org/10.1177/1461444814542311

[3] Moira Burke, Cameron Marlow, and Thomas Lento. 2010. Social network activity and social well-being. In Proceedings of the 28th international conference on Human factors in computing systems - CHI '10. ACM Press, Atlanta, Georgia, USA, 1909. https://doi.org/10.1145/1753326.1753613

[4] Paul Byron. 2019. 'How could you write your name below that?' The queer life and death of Tumblr. Porn Studies (June 2019), 1-14. https://doi.org/10.1080/ 23268743.2019.1613925

[5] Paul Byron. 2019. "Hey, I'm Having These Experiences": Tumblr Use and Young People's Queer (Dis)connections. (2019), 21.

[6] Matthew Carrasco and Andruid Kerne. 2018. Queer Visibility: Supporting LGBT+ Selective Visibility on Social Media. In Proceedings of the 2018 CHI Conference on Human Factors in Computing Systems - CHI '18. ACM Press, Montreal QC, Canada, 1-12. https://doi.org/10.1145/3173574.3173824

[7] Jenna L. Clark, Sara B. Algoe, and Melanie C. Green. 2018. Social Network Sites and Well-Being: The Role of Social Connection. Current Directions in Psychological Science 27, 1 (Feb. 2018), 32-37. https://doi.org/10.1177/0963721417730833

[8] Nicole B. Ellison, Jessica Vitak, Rebecca Gray, and Cliff Lampe. 2014. Cultivating Social Resources on Social Network Sites: Facebook Relationship Maintenance Behaviors and Their Role in Social Capital Processes. Journal of ComputerMediated Communication 19, 4 (July 2014), 855-870. https://doi.org/10.1111/jcc4. 12078

[9] Jesse Fox and Jennifer J. Moreland. 2015. The dark side of social networking sites: An exploration of the relational and psychological stressors associated with Facebook use and affordances. Computers in Human Behavior 45 (April 2015), 168-176. https://doi.org/10.1016/j.chb.2014.11.083

[10] Jesse Fox and Rachel Ralston. 2016. Queer identity online: Informal learning and teaching experiences of LGBTQ individuals on social media. Computers in Human Behavior 65 (Dec. 2016), 635-642. https://doi.org/10.1016/j.chb.2016.06.009

[11] Oliver L. Haimson, Jed R. Brubaker, Lynn Dombrowski, and Gillian R. Hayes. 2015. Disclosure, Stress, and Support During Gender Transition on Facebook. In Proceedings of the 18th ACM Conference on Computer Supported Cooperative Work \& Social Computing - CSCW' 15. ACM Press, Vancouver, BC, Canada, 1176-1190. https://doi.org/10.1145/2675133.2675152

[12] Ann Light. 2011. HCI as heterodoxy: Technologies of identity and the queering of interaction with computers. Interacting with Computers 23, 5 (Sept. 2011), 430-438. https://doi.org/10.1016/j.intcom.2011.02.002

[13] Alice E. Marwick and danah boyd. 2011. I tweet honestly, I tweet passionately: Twitter users, context collapse, and the imagined audience. New Media \& Society 13, 1 (Feb. 2011), 114-133. https://doi.org/10.1177/1461444810365313

[14] Elizabeth A. McConnell, Antonia Clifford, Aaron K. Korpak, Gregory Phillips, and Michelle Birkett. 2017. Identity, victimization, and support: Facebook experiences and mental health among LGBTQ youth. Computers in Human Behavior 76 (Nov. 2017), 237-244. https://doi.org/10.1016/j.chb.2017.07.026

[15] Ilan H. Meyer. 2003. Prejudice, social stress, and mental health in lesbian, gay, and bisexual populations: Conceptual issues and research evidence. Psychological Bulletin 129, 5 (Sept. 2003), 674-697. https://doi.org/10.1037/0033-2909.129.5.674

[16] Matthew B Miles, A Michael Huberman, Michael A Huberman, and Michael Huberman. 1994. Qualitative data analysis: An expanded sourcebook. sage.

[17] Brandon Miller. 2015. "They're the modern-day gay bar": Exploring the uses and gratifications of social networks for men who have sex with men. Computers in Human Behavior 51 (Oct. 2015), 476-482. https://doi.org/10.1016/j.chb.2015.05. 023

[18] Michal Pitoňák. 2017. Mental health in non-heterosexuals: Minority stress theory and related explanation frameworks review. Mental Health \& Prevention 5 (March 2017), 63-73. https://doi.org/10.1016/j.mhp.2016.10.002

[19] Morgan Klaus Scheuerman, Stacy M. Branham, and Foad Hamidi. 2018. Safe Spaces and Safe Places: Unpacking Technology-Mediated Experiences of Safety and Harm with Transgender People. Proceedings of the ACM on Human-Computer Interaction 2, CSCW (Nov. 2018), 1-27. https://doi.org/10.1145/3274424

[20] Katta Spiel, Alex Ahmed, Jennifer A. Rode, Jed R. Brubaker, Gopinaath Kannabiran, Os Keyes, Ashley Marie Walker, Michael A. DeVito, Jeremy Birnholtz, Emeline Brulé, Ann Light, Pınar Barlas, and Jean Hardy. 2019. Queer(ing) HCI: Moving Forward in Theory and Practice. In Extended Abstracts of the 2019 CHI Conference on Human Factors in Computing Systems - CHI EA '19. ACM Press, Glasgow, Scotland Uk, 1-4. https://doi.org/10.1145/3290607.3311750

[21] The Lancet. 2016. Meeting the unique health-care needs of LGBTQ people. The Lancet 387, 10014 (Jan. 2016), 95. https://doi.org/10.1016/S0140-6736(16)00013-1

[22] Chad Van De Wiele and Stephanie Tom Tong. 2014. Breaking boundaries: the uses \& gratifications of grindr. In Proceedings of the 2014 ACM International foint Conference on Pervasive and Ubiquitous Computing - UbiComp '14 Adjunct. ACM Press, Seattle, Washington, 619-630. https://doi.org/10.1145/2632048.2636070
[23] Erin A. Vogel, Jason P. Rose, Bradley M. Okdie, Katheryn Eckles, and Brittany Franz. 2015. Who compares and despairs? The effect of social comparison orientation on social media use and its outcomes. Personality and Individual Differences 86 (Nov. 2015), 249-256. https://doi.org/10.1016/j.paid.2015.06.026 